\title{
Interplay between Intestinal Microbiota and Host Immune System
}

\author{
Sarkis K. Mazmanian and Yun Kyung Lee \\ Division of Biology and Biological Engineering, California Institute of Technology, Pasadena, California, 91125, USA
}

Whether we are aware or not, diverse microorganisms are living on almost all environmentally exposed surfaces on our body without eliciting harmful immune responses. In fact, recent understanding from numerous studies indicates that our health is highly dependent on the contribution of intestinal commensal bacteria. It appears through its symbiotic interaction with the host, which is the result of millions of years of co-evolution, the microbiota shapes the immune system. In this review, we discuss the relationship between host physiology and commensal bacteria and explore the molecular mechanisms by which the adaptive immune system is influenced by the intestinal microbiota.

Key Words: Intestinal microbiota, Dysbiosis, Adaptive immune system, CD4+ T cells

\section{Introduction}

Bacteria have inhabited Earth for two billion years and populated almost every location on our planet. Although we have a little knowledge of how indigenous microorganisms began associating with the animals, including humans, it seems that the bacterial community adapted to colonize nutrient-rich niches within the host. In turn, the microbial ecosystem has been established in mammals to help digest complex carbohydrates and provide essential nutrients to mammals. To thrive within the host, the microbiota have evolved to play an irreplaceable role in the host health for their mutual benefit. This relationship between microbiota and their host could be viewed by a term of symbiosis and the microbes are called as symbionts. In contrast, certain commensal microbes, while possibly providing some benefits to the host homeostatic conditions, may cause diseases under certain genetic and environmental conditions. These microbes have been termed as pathobionts $(1,2)$. It is widely accepted that the microbiota contains a balanced composition of symbionts and pathobionts under normal condition. An unnatural shift in the composition of the microbiota, called dysbiosis, was observed in many other diseases as well as autoimmune diseases (3). Western societies display a decreased prevalence of many infectious diseases that is a result of improved hygienic practices as well as the development of antibiotics and vaccination. Interestingly, there is obvious increase in the incidence of non-infectious immune disorders such as autoimmune diseases and allergic responses (4). This inverse correlation implies that the environmental changes resulting in a disruption of commensal microbial communities may be a potential cause of dysregulation of immune system.

The immune response is charged with the responsibility to distinguish between self and non-self. With the fact that numerous microorganisms are colonized on variable surfaces of the host, host immune system has been evolutionally forced to develop the ability to distinguish between commensal microbiota (considered as self) and pathogens.

Received: January 6, 2014/ Revised: January 8, 2014/ Accepted: January 10, 2014

* Corresponding author: Yun Kyung Lee. Division of Biology, California Institute of Technology, Pasadena, California, 91125, USA.

Phone: +1-626-395-8980, Fax:+1-626-395-2484, e-mail: bioyk@caltech.edu

(c) This is an Open Access article distributed under the terms of the Creative Commons Attribution Non-Commercial License (http://creativecommons.org/license/by-nc/3.0/). 
The host has evolved several mechanisms to obtain immunological ignorance to commensals. The production of thick mucus layer that overlie the intestinal epithelium is the first barrier to prevent the access of microbes (5). Symbiotic bacteria promote the secretion of antimicrobial proteins by gut epithelial cells for limiting bacteria penetration across the epithelial barrier $(5,6)$. Intestinal dendritic cells (DCs) can retain small numbers of live commensals, which allow DCs to selectively induce immunoglobulin A (IgA) and help sequestering enteric bacteria in the gut lumen (7). The luminal compartmentalization (the mucus barrier, antimicrobial proteins and secretory $\operatorname{IgA}$ ) is an essential determinant of symbiotic host-microorganism relationships to avoid over-activation of immune response against commensals (5). Colonization resistance is another mechanism that the intestinal microbiota protects itself by establishing the relatively stable microbial ecosystem to resist subsequent colonization by invading foreign microbes (8). Moreover, the development of the adaptive immune system allows for further pathogen discrimination and maintenance of mucosal homeostasis. Interestingly, recent studies suggest that commensal microbiota have actively involved in the development of adaptive immune responses by programming many aspects of $\mathrm{CD} 4+\mathrm{T}$ cell differentiation, that extends beyond regulating microbial communities. This article will review relevant studies about the effect of the microbiota on human health and the role of the microbiota on the development of adaptive immune responses. The understanding of these phenomena may lead us to develop novel therapy to prevent and cure numerous immunologic diseases.

\section{Understanding the human microbiota}

We encounter the tremendously diverse microbial communities over the course of our lives starting with a birth. Immediately after birth, newborns are exposed to and rapidly colonized by microbes from the environment. The microbial diversity in the infant gut is initially very low and then is dramatically increased depending on dietary shift and differential exposed environment over the first few years of life (9). Colonization and diversification occur within time in the various microbial habitats of the body and the differences in the microbiota composition exist among individuals (9). It is thought that during this period the host immune system is learning to distinguish between commensal and pathogenic microbes. In adults, the stable microbiota are assembled depending on body surfaces and then homeostasis is achieved by the symbiotic interaction between host and microorganisms. The microbial ecosystem in the body is dynamic in response to external environment to maintain homeostasis. The disruption of these microbial communities may lead to the dysregulation of the homeostasis between humans and their commensals and possibility alter disease susceptibility.

Although the microbiota has long been considered as a critical factor for human health, technologies in the laboratory were limited to cultivatable bacteria, which are a minority of the microbiota. The development of molecular profiling methods, including high-throughput sequencing of microbial $16 \mathrm{~S}$ ribosomal RNA genes have allowed complex microbial communities to be characterized more comprehensively. Recent efforts such as the Metagenomics of the Human Intestinal Tract (MetaHIT) consortium and Human Microbiome Project (HMP) have revealed the majority of the microbiota in several anatomical locations including skin, mouth, intestine and urogenital tract of our bodies by culture-independent analysis $(10,11)$. The knowledge from these trials provides insights about human microbiome composition, carrying implications into host-microbial and microbial-microbial interactions. Several signature findings from these projects were observed and a map of human microbiome diversity in particular body sites was generated (12). The gastrointestinal tract is the place for the most abundant and various microbiota among our body habitats. Mammalian host harbors average more than $10^{11} \sim 10^{12}$ microbial cells per gram colonic content and composed of more than 1000 distinct bacterial species (13). The composition of the gut microbiota considerably varies between individuals. The human intestinal microbiota is similar to the microbiota of other mammals at the phylum level but distinct at the species and strain levels (12). The most 
predominant intestinal phyla are the Bacteroidetes and the Firmicutes $(10,12)$. The intestinal Firmicutes are Grampositive bacteria that are consisting of species the Clostridia class, Enterococcaceae and Lactobacillaceae families. Intestinal Bacteroidetes are Gram-negative bacteria comprised of several Bacteroides species. The relative proportions of these two phyla in the gastrointestinal tract have been shown to strongly correlate with states of human health and disease.

\section{The microbiota in diseases-studies from model systems}

Germ-free (GF) animals are born and raised in the absence of microbes, which allows for insights into the impact of the commensal microbiota on host health (14). Studies of GF mice have shown that the gut microbiota affects the metabolism of nutrients, neurologic responses and the development of immune system. GF animals require $30 \%$ more calories to maintain their body weight, comparing those with the microbiota (15). It indicates that the gut microbiota is necessary for host metabolism and digestive efficiency. Recent reports demonstrate that GF mice display increased motor activity and reduced anxiety-like behavior, suggesting the microbiota modulates brain development and behavior (16). Most of all, GF animals have severe defects in the development of gut-associated lymphoid tissue (GALT). GF mice display developmental defects in tissue formation including: fewer and smaller Peyer's patches, reduced number of IgA-secreting plasma cells, and smaller mesenteric lymph nodes $(13,17)$. Intestinal epithelial cells express reduced expression of Toll-like receptors (TLRs) and class II major histocompatibility complex (MHCII) molecules in the absence of the microbiota (13, 17). Moreover, GF mice also display reduced numbers of $\mathrm{CD} 4+\mathrm{T}$ cells in the lamina propria and spleen. The microbiota also influences pathogen clearance, so GF mice are more susceptible to infectious agents such as Citrobacter rodentium and Campylobacter jejuni (18). Therefore, the contribution of the microbiota to the development and function of the immune system appear to be fundamental.

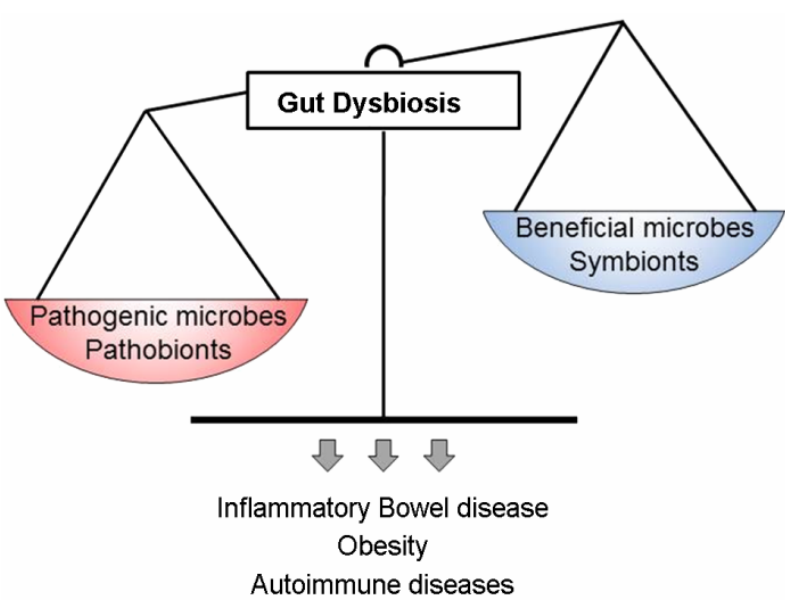

Figure 1. Gut dysbiosis are associated with human diseases. The microbiota in the mammal gastrointestinal tract can divide to pathogenic and beneficial bacteria called pathobionts and symbionts respectively. A healthy microbiota contains a balanced composition of pathobionts and symbionts. If there is an unnatural shift in the composition of the gut microbiota, gut dysbiosis, can lead to intestinal inflammation, many other diseases and autoimmune diseases.

In addition to its roles in the development of host immune responses, the intestinal microbiota influences on the development of many other disorders. Dysbiosis of gut microbial communities is observed in several diseases such as gastrointestinal diseases, obesity and autoimmune diseases (Fig. 1). The compositional change of the gut microbiota was observed in patients with inflammatory bowel disease (IBD), although this change could be either the cause or the result of aberrant inflammation $(13,19)$. It is widely accepted that human IBD is not caused by a specific pathogen, but by an immune system imbalance that is associated with gut dysbiosis. In animal studies, the transfer of microorganisms from IBD mice was sufficient to induce experimental IBD $(20,21)$. Recent interesting studies have shown that western-diet can be one of the inducing factors for dysbiosis. Dietary fat influences the composition and complexity of the gut microbiota by favoring the growth of a pathobiont, Bilophila wadsworthia (22). It contributes to induce the development of inflammatory bowel disease in genetically susceptible animals (22). Dysbiosis has been also implicated in the development of extra-intestinal disorders. The altered composition of the gut microbiota, which leads 
to altered fermentation and increased energy harvest, was reported in obese animals. Studies have shown that obese mice have more Firmicutes and fewer Bacteroidetes than genetically lean siblings (23). When GF mice are colonized with the microbiota from obese mice, they gained more mean body fat than with the microbiota from lean mice demonstrating the transmissibility of metabolic phenotype (23). Consistent with this observation, the proportion of Bacteroidetes in the microbiota of obese people is lower than that of lean people (24). The contribution of the microbiota to promote autoimmune diseases, which are mostly caused by the dysregulation of adaptive immune system, has been studied by animal models. It appears that the colonization of segmented filamentous bacteria (SFBs), a pathobiont, leads to the development of autoimmune arthritis $(\mathrm{K} / \mathrm{BxN}$ mouse model) and experimental autoimmune encephalomyelitis (EAE; an animal model for multiple sclerosis) $(25,26)$. It clearly shows that alterations in the normal intestinal microbiota can affect systemic immune-mediated diseases.

\section{The microbiota and microbial metabolites-the function of CD4+ $\mathrm{T}$ cells}

\section{Anti-inflammatory roles of the microbiota and metabolites; Treg cells}

Regulatory T cells (Tregs) are critical towards maintaining immune balance and restricting aberrant inflammation (13, 17). A human commensal, Bacteroides fragilis, has revealed its ability to induce IL-10, an anti-inflammatory cytokine, production among Tregs in the colon (27). B. fragilis expresses a capsular polysaccharide A (PSA) which directs Treg development to prevent inflammation in experimental models of colitis and $\operatorname{EAE}(28,29)$. It appears that PSA induces the production of TGF $\beta 2$ and acts directly on CD4+ T cells by TLR 2 signaling $(27,30)$. Among the indigenous microbiota in the murine colon, the genus Clostridium clusters IV and XIVa are also reported as an inducer of colonic Tregs. The colonization of GF mice with a defined mixture of 46 strains of Clostridium from conventional mice sufficiently induces Tregs (31). Although the determinant of Clostridia inducing Tregs is not clear, Clostridium species influence the differentiation of Tregs via epithelial cellderived TGF $\beta 1$ (31). The effect of Clostridium was independent of several bacterial associated pattern recognition receptors and Treg cells induction occurred in mice deficient in MyD88 (a signaling adaptor molecule for Toll-like receptors), Rip2 (an adaptor molecule for NOD receptors) and Card9 (a key transducer of Dectin-1 signaling)-mediated signaling pathways (31). In similar mechanism, 17 strains within clusters IV, XIVa and XVIII of Clostridia provide bacterial antigens and TGF $\beta$ rich environment to help expansion and differentiation of Treg cells in human (32). Therefore, it indicates that genera from disparate phyla have evolved distinct mechanisms to influence the development of Treg cells (Fig. 2).

It has long been appreciated that commensal bacteria are necessary for digestion of fibers. The breakdown and fermentation of polysaccharides into short-chain fatty acids (SCFAs) is a reserved role by the microbiota despite the microbiota variability between different individuals. $5 \sim 10 \%$ of the total calorie requirement of humans is dependent on the SCFAs produced by indigenous bacteria (33). Recent reports have found the molecular mechanisms that SCFAs (acetic acid, propionic acid and butyric acid) mediates development of Treg cells in the intestine, although the mechanism by these three SCFAs is slightly different. The administration of acetate and propionate increases total number of Treg cells in the colon, which is mediated by the signaling of G-protein coupled receptors (GPR) 43 (34). Butyrate leads to the generation of colonic Tregs from naïve CD4+ T cells by the inhibition of histone deacetylase (HDAC) (Fig. 2) (35 37). The orally administrated SCFAs confer protection in $\mathrm{T}$ cell dependent colitis models $(34,35)$. Given the production of SCFAs by Clostiridia colonization was observed, it is highly possible that these bacteria might be using SCFAs-mediated mechanism to induction of Tregs in the intestine (35). Intestinal bacterial metabolites and microbial components are an important communication tool between the host immune system and the microbiota to establish mutualism. 


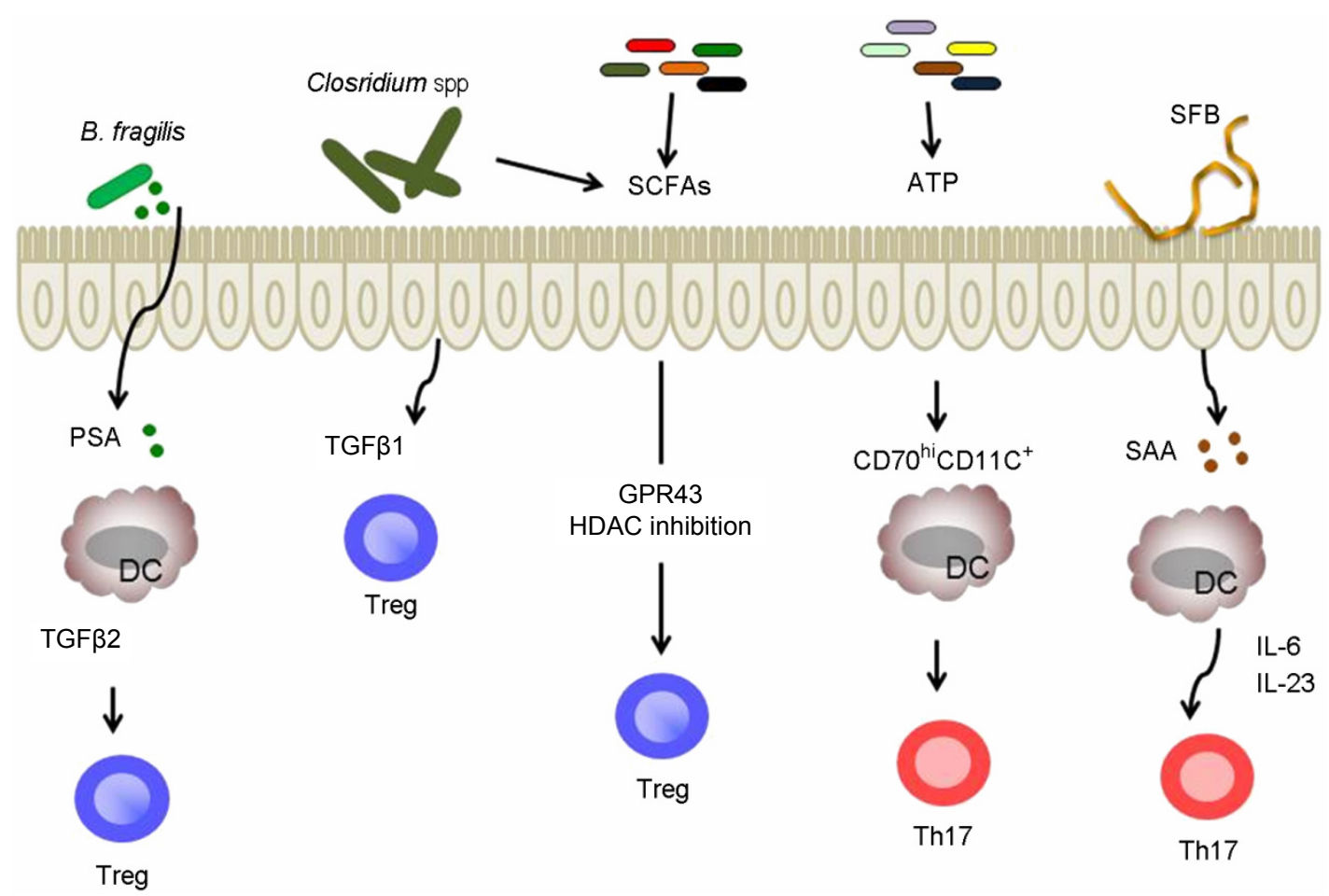

Figure 2. Immunomodulatory members of the gut microbiota and microbial metabolites regulate the function of CD4+ T cell subsets. B. fragilis or Clostridium spp. promotes the induction of Tregs in colonic laminar propria via PSA-TLR2 signaling or the secretion of TGF $\beta 1$ respectively. SCFAs mediate the proliferation and induction of Treg cells in colon through distinct mechanism; GPR43 or HDAC inhibitory activity. Commensal bacteria derived-extracellular ATP induces the differentiation of Th17 cells in colon. Colonization of SFBs promotes the development of Th17 cells in laminar propria of small intestine by inducing the production of SAA.

\section{The microbiota and metabolites inducing the effector immune responses; Th17 cells}

IL-17 producing effector CD4+ T cells (Th17) are important for the protection against extracellular pathogens and dysregulation of Th17 cells promotes the development of inflammatory disorders and autoimmune diseases (13, 17). Extracellular adenosine 5'-triphosphate (ATP) is known to induce the differentiation of Th17 cells in intestinal laminar propria (38). ATP is a product of the intestinal microbiota activity, as there are reduced fecal levels of ATP in GF mice and high ATP concentrations were detected in the intestinal contents of conventionally colonized mice. The addition of the supernatants of in vitro cultured intestinal commensal bacteria enhances the differentiation of Th17 cells, which is inhibited by the presence of the ATP degrading enzyme (38). It seems that intestinal lamina propria $\mathrm{CD} 70^{\mathrm{hi}} \mathrm{CD} 11 \mathrm{C}^{+}$cells preferentially induce $\mathrm{Th} 17$ cells in response to ATP stimulation (Fig. 2) (38). Thereby, extracellular ATP is one of the factors to induce Th17 cells derived from commensals, although it is not likely ATP is produced by certain specific microorganism.

SFBs are Gram-positive anaerobic bacteria with highly reduced genome $(39,40)$. The complete genome of SFBs shows that some metabolic functions are highly dependent on the host and it explains why these bacteria cannot be cultured. SFBs are specifically colonized in small intestine starting at the time of weaning $(41,42)$. Physical association with intestinal epithelial cells makes SFBs highly effective in stimulating the mucosal immune system (43). Colonization of GF mice with SFBs promotes the production of antimicrobial peptides, pro-inflammatory cytokines, and IgA secretion by B cells (44). Most interestingly, SFBs specifically induce IL-17 producing CD4+ T cells in terminal 
ileum $(45,46)$. Intestinal colonization with SFBs changes the several gene expression profiles of the host cells in the intestine. It appears that serum amyloid A (SAA) induced by SFBs in the terminal ileum can stimulate intestinal DCs to produce IL-6 and IL-23, in which both cytokines are important for Th17 cell differentiation (45). SFB-mediated Th17 cell differentiation occurs through a mechanism independent of ATP signaling (45) (Fig. 2). SFB-colonized mice can restrict infection by murine pathogen $C$. rodentium, suggesting these bacteria participate in the barrier function (45). Given SFBs are endowed with pro-inflammatory properties by inducing Th17 cells, SFBs have emerged as a pathobionts model system. The outcome of SFBs-host interactions depends on the immune status or genetic predisposition of the host, as SFBs have been associated with the development of intestinal inflammation and autoimmune diseases $(25,26)$. Together with a defined microbiota mixture, SFBs can induce colitis in animal models (47). It also reported that the colonization with SFBs promotes the development of autoimmune arthritis and $\operatorname{EAE}(25,26)$. Therefore, particular subset of bacteria from commensal influences systemic immune responses, not only gut immune responses. Further investigations are needed to establish the molecular basis of SFBs-mediated Th17 differentiation.

\section{Probiotics}

The term probiotics is used to describe microorganisms that provide health benefits to host when consumed by diet. The induction of Tregs by probiotics is highlighted by the amelioration of inflammation and diseases, although it has an effect on several cell types including epithelial cells, DCs and T cells. It is reported that Bifidobacterium breve can induce IL-10 producing CD4+ T cells in the colon by activating intestinal colonic $\mathrm{CD} 103^{+} \mathrm{DC}$ s to produce $\mathrm{IL}-10$ via the TLR2-MyD88 pathway (48). Treatment of mice with the probiotic mixture VSL\#3 (a mixture of Bifidobacteria, Lactobacilli and Streptococcus salivarius) or the probiotic strain Lactobacillus reuteri increases the percentage of Tregs in colon (49). The administration of these probiotics has an effect on suppressing intestinal inflammation in several experimental colitis models $(48,49)$. It seems that the probiotics also has protective effects on allergic responses in lungs and alleviates the symptom of atopic dermatitis in animal model, although these effects have not been demonstrated in human studies $(50,51)$. Therefore, further studies to define molecular mechanism are required for medical practice or therapy.

\section{Conclusions}

We are just now beginning to understand the nature of our relationship with these microbes, making this an exciting time for human microbiota studies. These studies will include large scale techniques that are capable to do both basic biological studies and translational studies. More intensive investigations are now required to understand the interaction between microbiota and human health in many ways. The first step is the understanding stable and diverse microbial community within an individual through time and it can lead to identify personalized human microbiome. The studies to understand the molecular mechanism of the microbiota to regulate host immune system are also needed. These insights may enable the prediction of disease onset and progression and develop therapy, when the disturbance events such as diet shifts, antibiotic use, surgery, drug treatments or transplantations perturb the microbiota humanmicrobe relationship. It can lead to manipulate and reconstitution of the microbiota to re-establish healthy and stable microbial community.

\section{REFERENCES}

1) Chow J, Mazmanian SK. A pathobiont of the microbiota balances host colonization and intestinal inflammation. Cell Host Microbe 2010;7:265-76.

2) Chow J, Tang H, Mazmanian SK. Pathobionts of the gastrointestinal microbiota and inflammatory disease. Curr Opin Immunol 2011;23:473-80.

3) Cho I, Blaser MJ. The human microbiome: at the interface of health and disease. Nat Rev Genet 2012; 13:260-70.

4) Bach JF. The effect of infections on susceptibility to 
autoimmune and allergic diseases. N Engl J Med 2002; 347:911-20.

5) Hooper LV, Macpherson AJ. Immune adaptations that maintain homeostasis with the intestinal microbiota. Nat Rev Immunol 2010;10:159-69.

6) Vaishnava S, Yamamoto M, Severson KM, Ruhn KA, $\mathrm{Yu} \mathrm{X}$, Koren $\mathrm{O}$, et al. The antibacterial lectin RegIIIgamma promotes the spatial segregation of microbiota and host in the intestine. Science 2011;334: 255-8.

7) Macpherson AJ, Uhr T. Induction of protective IgA by intestinal dendritic cells carrying commensal bacteria. Science 2004;303:1662-5.

8) Buffie CG, Pamer EG. Microbiota-mediated colonization resistance against intestinal pathogens. Nat Rev Immunol 2013;13:790-801.

9) Clemente JC, Ursell LK, Parfrey LW, Knight R. The impact of the gut microbiota on human health: an integrative view. Cell 2012;148:1258-70.

10) Turnbaugh PJ, Ley RE, Hamady M, Fraser-Liggett CM, Knight R, Gordon JI. The human microbiome project. Nature 2007;449:804-10.

11) Dethlefsen L, McFall-Ngai M, Relman DA. An ecological and evolutionary perspective on humanmicrobe mutualism and disease. Nature 2007;449:811-8.

12) Morgan $X C$, Segata N, Huttenhower C. Biodiversity and functional genomics in the human microbiome. Trends Genet 2013;29:51-8.

13) Round JL, Mazmanian SK. The gut microbiota shapes intestinal immune responses during health and disease. Nat Rev Immunol 2009;9:313-23.

14) Faith JJ, Rey FE, O'Donnell D, Karlsson M, McNulty NP, Kallstrom G, et al. Creating and characterizing communities of human gut microbes in gnotobiotic mice. ISME J 2010;4:1094-8.

15) Wostmann BS, Larkin C, Moriarty A, Bruckner-Kardoss E. Dietary intake, energy metabolism, and excretory losses of adult male germfree Wistar rats. Lab Anim Sci 1983;33:46-50.

16) Diaz Heijtz R, Wang S, Anuar F, Qian Y, Björkholm B, Samuelsson A, et al. Normal gut microbiota modulates brain development and behavior. Proc Natl Acad Sci U S A 2011;108:3047-52.

17) Lee YK, Mazmanian SK. Has the microbiota played a critical role in the evolution of the adaptive immune system? Science 2010;330:1768-73.

18) Lupp C, Robertson ML, Wickham ME, Sekirov I, Champion OL, Gaynor EC, et al. Host-mediated inflammation disrupts the intestinal microbiota and promotes the overgrowth of Enterobacteriaceae. Cell Host Microbe 2007;2:119-29.

19) Joossens M, Huys G, Cnockaert $M$, De Preter V, Verbeke K, Rutgeerts $\mathrm{P}$, et al. Dysbiosis of the faecal microbiota in patients with Crohn's disease and their unaffected relatives. Gut 2011;60:631-7.

20) Elinav E, Strowig T, Kau AL, Henao-Mejia J, Thaiss CA, Booth CJ, et al. NLRP6 inflammasome regulates colonic microbial ecology and risk for colitis. Cell 2011; 145:745-57.

21) Garrett WS, Lord GM, Punit S, Lugo-Villarino G, Mazmanian SK, Ito S, et al. Communicable ulcerative colitis induced by T-bet deficiency in the innate immune system. Cell 2007;131:33-45.

22) Devkota S, Wang Y, Musch MW, Leone V, FehlnerPeach H, Nadimpalli A, et al. Dietary-fat-induced taurocholic acid promotes pathobiont expansion and colitis in Il10-/- mice. Nature 2012;487:104-8.

23) Turnbaugh PJ, Ley RE, Mahowald MA, Magrini V, Mardis ER, Gordon JI. An obesity-associated gut microbiome with increased capacity for energy harvest. Nature 2006;444:1027-31.

24) Ley RE, Turnbaugh PJ, Klein S, Gordon JI. Microbial ecology: human gut microbes associated with obesity. Nature 2006;444:1022-3.

25) Wu HJ, Ivanov II, Darce J, Hattori K, Shima T, Umesaki $\mathrm{Y}$, et al. Gut-residing segmented filamentous bacteria drive autoimmune arthritis via $\mathrm{T}$ helper 17 cells. Immunity 2010;32:815-27.

26) Lee YK, Menezes JS, Umesaki Y, Mazmanian SK. Proinflammatory T-cell responses to gut microbiota promote experimental autoimmune encephalomyelitis. Proc Natl Acad Sci U S A 2011;108 Suppl 1:4615-22.

27) Round JL, Mazmanian SK. Inducible Foxp3+ regulatory T-cell development by a commensal bacterium of the intestinal microbiota. Proc Natl Acad Sci U S A 2010; 107:12204-9.

28) Mazmanian SK, Round JL, Kasper DL. A microbial symbiosis factor prevents intestinal inflammatory disease. 
Nature 2008;453:620-5.

29) Ochoa-Repáraz J, Mielcarz DW, Ditrio LE, Burroughs AR, Begum-Haque S, Dasgupta S, et al. Central nervous system demyelinating disease protection by the human commensal Bacteroides fragilis depends on polysaccharide A expression. J Immunol 2010;185:4101-8.

30) Round JL, Lee SM, Li J, Tran G, Jabri B, Chatila TA, et al. The Toll-like receptor 2 pathway establishes colonization by a commensal of the human microbiota. Science 2011;332:974-7.

31) Atarashi K, Tanoue T, Shima T, Imaoka A, Kuwahara T, Momose $\mathrm{Y}$, et al. Induction of colonic regulatory $\mathrm{T}$ cells by indigenous Clostridium species. Science 2011; 331:337-41.

32) Atarashi K, Tanoue T, Oshima K, Suda W, Nagano Y, Nishikawa $\mathrm{H}$, et al. Treg induction by a rationally selected mixture of Clostridia strains from the human microbiota. Nature 2013;500:232-6.

33) Mortensen PB, Clausen MR. Short-chain fatty acids in the human colon: relation to gastrointestinal health and disease. Scand J Gastroenterol Suppl 1996;216:132-48.

34) Smith PM, Howitt MR, Panikov N, Michaud M, Gallini CA, Bohlooly-Y M, et al. The microbial metabolites, short-chain fatty acids, regulate colonic Treg cell homeostasis. Science 2013;341:569-73.

35) Furusawa Y, Obata Y, Fukuda S, Endo TA, Nakato G, Takahashi D, et al. Commensal microbe-derived butyrate induces the differentiation of colonic regulatory $\mathrm{T}$ cells. Nature 2013;504:446-50.

36) Arpaia N, Campbell C, Fan X, Dikiy S, van der Veeken $\mathrm{J}$, deRoos $\mathrm{P}$, et al. Metabolites produced by commensal bacteria promote peripheral regulatory T-cell generation. Nature 2013;504:451-5.

37) Chang PV, Hao L, Offermanns S, Medzhitov R. The microbial metabolite butyrate regulates intestinal macrophage function via histone deacetylase inhibition. Proc Natl Acad Sci U S A 2014.

38) Atarashi K, Nishimura J, Shima T, Umesaki Y, Yamamoto M, Onoue $\mathrm{M}$, et al. ATP drives lamina propria $\mathrm{T}(\mathrm{H}) 17$ cell differentiation. Nature 2008;455: 808-12.

39) Sczesnak A, Segata N, Qin X, Gevers D, Petrosino JF, Huttenhower $\mathrm{C}$, et al. The genome of th17 cell-inducing segmented filamentous bacteria reveals extensive auxotrophy and adaptations to the intestinal environment. Cell Host Microbe 2011;10:260-72.

40) Prakash T, Oshima K, Morita H, Fukuda S, Imaoka A, Kumar $\mathrm{N}$, et al. Complete genome sequences of rat and mouse segmented filamentous bacteria, a potent inducer of th17 cell differentiation. Cell Host Microbe 2011;10:273-84.

41) Blumershine RV, Savage DC. Filamentous microbes indigenous to the murine small bowel: A scanning electron microscopic study of their morphology and attachment to the epithelium. Microb Ecol 1977;4:95 -103 .

42) Koopman JP, Stadhouders AM, Kennis HM, De Boer $\mathrm{H}$. The attachment of filamentous segmented microorganisms to the distal ileum wall of the mouse: a scanning and transmission electron microscopy study. Lab Anim 1987;21:48-52.

43) Cerf-Bensussan N, Gaboriau-Routhiau V. The immune system and the gut microbiota: friends or foes? Nat Rev Immunol 2010;10:735-44.

44) Gaboriau-Routhiau V, Rakotobe S, Lécuyer E, Mulder I, Lan A, Bridonneau C, et al. The key role of segmented filamentous bacteria in the coordinated maturation of gut helper T cell responses. Immunity 2009;31:677-89.

45) Ivanov II, Atarashi K, Manel N, Brodie EL, Shima T, Karaoz U, et al. Induction of intestinal Th17 cells by segmented filamentous bacteria. Cell 2009;139:485-98.

46) Ivanov II, Frutos Rde L, Manel N, Yoshinaga K, Rifkin $\mathrm{DB}$, Sartor RB, et al. Specific microbiota direct the differentiation of IL-17-producing T-helper cells in the mucosa of the small intestine. Cell Host Microbe 2008; 4:337-49.

47) Stepankova R, Powrie F, Kofronova O, Kozakova H, Hudcovic T, Hrncir T, et al. Segmented filamentous bacteria in a defined bacterial cocktail induce intestinal inflammation in SCID mice reconstituted with CD45RBhigh CD4+ T cells. Inflamm Bowel Dis 2007; 13:1202-11.

48) Jeon SG, Kayama H, Ueda Y, Takahashi T, Asahara T, Tsuji H, et al. Probiotic Bifidobacterium breve induces IL-10-producing Tr1 cells in the colon. PLoS pathog 2012;8:e1002714.

49) Di Giacinto C, Marinaro M, Sanchez M, Strober W, Boirivant M. Probiotics ameliorate recurrent Th1- 
mediated murine colitis by inducing IL-10 and IL-10dependent TGF-beta-bearing regulatory cells. J Immunol 2005; 174:3237-46.

50) Lyons A, O'Mahony D, O'Brien F, MacSharry J, Sheil $\mathrm{B}$, Ceddia $\mathrm{M}$, et al. Bacterial strain-specific induction of Foxp $3+\mathrm{T}$ regulatory cells is protective in murine allergy models. Clin Exp Allergy 2010;40:811-9.

51) Kim NY, Ji GE. Effects of probiotics on the prevention of atopic dermatitis. Korean J Pediatr 2012;55:193-201. 